\title{
Datos sobre el peso al nacer en países en desarrollo: ¿son útiles las encuestas?'1
}

\author{
J. T. Boerma, ${ }^{2}$ K. I. Weinstein, ${ }^{3}$ S. O. Rutstein ${ }^{4}$ \\ y A. E. Sommerfelt ${ }^{5}$
}

RESUMEN Las estadísticas de centros de atención de salud son la fuente principal de datos sobre el peso al nacer en los países en desarrollo, si bien en la mayor parte de esos países no se producen estimaciones anuales de la incidencia de bajo peso al nacer a partir de esos datos. Si se produjeran, las estimaciones serían propensas al sesgo de selección ya que los datos están limitados en general a los niños nacidos en centros de salud $y$, por lo tanto, representan un subgrupo decididamente diferente de la población general de neonatos. Desde 1990, el programa de Encuestas Demográficas y de Salud ha realizado 15 encuestas nacionales en las que se incluyeron preguntas sobre el peso y el tamaño relativo al nacer tal como los recordaban las madres. Este artículo muestra que las encuestas transversales pueden constituir una fuente útil de datos para estimar la media nacional de peso al nacer y la incidencia de bajo peso. Sin embargo, la clasificación errónea del peso al nacer es demasiado extensa para emplear los datos de tamaño relativo al nacer como indicador de bajo peso a escala individual.

La información sobre el peso al nacer es importante por las siguientes razones: las estimaciones nacionales y regionales de la incidencia de bajo peso al nacer (BPN) se reconocen a nivel internacional como indicadores del bienestar de los neonatos y de las mujeres en edad fecunda; dichas estimaciones proveen información pun-

1 Se publicó en inglés en el Bulletin of the World Health Organization, 1996, Vol. 74, No. 2, pp. 209-216, con el título "Data on birth weight in developing countries: can surveys help?" @) Organización Mundial de la Salud, 1996.

2 Proyecto TANESA, Mwanza, Tanzanía. La correspondencia y pedidos de separatas deben dirigirse a este autor a la siguiente dirección: TANESA Project, P.O. Box 434, Mwanza, República Unida de Tanzanía.

3 Consultor independiente, Medford, Nueva Jersey, Estados Unidos de América.

4 Macro International, Encuestas Demográficas y de Salud, Dirección del Análisis, Calverton, Maryland, Estados Unidos de América.

5 Macro International, Encuestas Demográficas y de Salud, Análisis de Salud. tual sobre la salud de las embarazadas y la evolución del desarrollo fetal y, como es bien sabido, el peso al nacer es uno de los factores principales que determinan la probabilidad que tiene un neonato de sobrevivir (1). Recientemente se seleccionó la incidencia de BPN como uno de los indicadores empleados para monitorear las metas de salud establecidas en la Cumbre Mundial en Favor de la Infancia; en este caso, para reducir a $10 \%$ o menos la incidencia de BPN (definido como $<2500 \mathrm{~g}$ ).

Las estadísticas de los centros de salud son la fuente principal de datos sobre el peso al nacer en los países en desarrollo. La mayoría de esos países no producen estimaciones anuales de la incidencia de BPN a partir de dichos datos. En muchos países, la mayoría de los niños no nacen en centros de salud; de hecho, más de tres cuartas partes de los nacimientos recientes en Indonesia, Marruecos, Nigeria, Pakistán y Yemen tuvieron lugar en el hogar (2-6). Entonces, las estimaciones limitadas a niños nacidos en centros de atención sanitaria estarían sujetas a sesgo de selección, pues hay buenas razones para suponer que esos recién nacidos son muy diferentes del resto de la población. Con toda probabilidad, la incidencia de BPN se subestimaría y las conclusiones resultantes sobre el riesgo de bajo peso al nacer serían equívocas.

Otra fuente de información sobre el peso al nacer son los datos retrospectivos obtenidos de encuestas. Da Vanzo et al. mostraron que los datos retrospectivos de la Encuesta sobre la Vida Familiar en Malasia (7) podían usarse para investigar los correlatos del BPN, pero que las inferencias deducidas de pesos al nacer recordados resultan ses- 
gadas. Al analizar la Encuesta Demográfica y de Salud del Perú, Moreno y Goldman (8) mostraron que los datos retrospectivos podían usarse para estimar la incidencia de BPN y concluyeron que las estimaciones de peso al nacer basadas en los pesos recordados estaban claramente subestimadas. Esos análisis impulsaron al Demographic and Health Surveys programme [programa de Encuestas Demográficas y de Salud] (DHS) a incluir en las encuestas nacionales preguntas para las madres sobre el peso y el tamaño relativo al nacer de sus hijos. En este artículo se trata de determinar si las encuestas transversales pueden aprovecharse para mejorar las estimaciones nacionales de la media del peso al nacer y la incidencia de BPN. Además, se explora la idea de utilizar los datos sobre el tamaño al nacer para hacer análisis de escala individual, tales como los estudios de las causas que determinan la mortalidad infantil, de la antropometría infantil y de los efectos de la atención materna.

\section{MATERIALES Y MÉTODOS}

El DHS, que recibe fondos de la Agencia de los Estados Unidos para el Desarrollo Internacional (AID), ayuda a los países en desarrollo a organizar encuestas nacionales representativas, que proporcionan información para fundamentar la adopción de decisiones sobre políticas y programas, y la investigación científica. La información requerida en la encuesta incluye una historia completa de los nacimientos, la cual se emplea para estimar la fecundidad, la mortalidad infantil y varios indicadores de la salud materna y la infantil. La encuesta está dirigida a las mujeres en edad de procrear.

Desde 1990, las encuestas del DHS incluyen la siguiente serie de preguntas relacionadas con todos los niños nacidos durante los 5 años anteriores:

- Cuando (nombre) nació, ¿era muy grande, más grande que el término medio, de término medio, más pequeño(a) que el término medio o muy pequeño(a)?
- ¿Se pesó a (nombre) al nacer?

- (Si la respuesta es afirmativa) ¿Cuánto pesó (nombre)?

Se analizaron los datos obtenidos de las 15 encuestas que contenían esas preguntas. En 12 de las 15, la pregunta sobre el tamaño relativo al nacer se hizo tal como se indica arriba. En Camerún y Yemen se combinaron las categorías "muy grande" y "más grande que el término medio". En Paraguay se usaron solo tres categorías: "grande", "de término medio" y "pequeño(a)". En todas las encuestas se les hizo a las madres una pregunta sobre el tamaño relativo al nacer de cada uno de los niños, incluso cuando habían proporcionado un peso al nacer numérico.

La utilidad de los datos de encuestas sobre el peso al nacer depende de la capacidad de las madres para recordar con precisión el peso al nacer de los niños que fueron pesados en ese momento y de la calidad de los datos sobre el tamaño relativo al nacer. Dado que en los Estados Unidos se mantiene un sistema preciso de registro de las estadísticas vitales que incluye el peso al nacer anotado en los certificados de nacimiento, Ekouevi y Morgan (9) pudieron evaluar la validez de la información retrospectiva sobre peso al nacer en las USA National Surveys of Family Growth [Encuestas Nacionales de los EUA sobre el Crecimiento de las Familias] comparando los datos recordados con los consignados en el sistema de registro. También pudieron sopesar la confiabilidad de la información retrospectiva del peso al nacer comparando los datos recordados en varias encuestas. Así se descubrió que las frecuencias y tendencias de las estimaciones de BPN basadas en datos retrospectivos coincidían en general con los datos del sistema de registro y que las frecuencias y tendencias estimadas eran similares de una encuesta a otra. Estos dos hallazgos confirman en principio la confiabilidad de la información retrospectiva sobre el peso al nacer. La calidad de los datos numéricos sobre peso al nacer de las encuestas del DHS solo puede evaluarse comparando los resultados con los de otros estudios nacionales del mismo país. Sin embargo, esos estudios están basados en datos hospitalarios y no suelen cubrir períodos concurrentes.

La calidad de los datos sobre el tamaño relativo al nacer puede evaluarse solamente en función de los nacimientos para los cuales se cuenta con datos numéricos. La media del peso al nacer y la incidencia de BPN deben corresponder al tamaño relativo al nacer recordado. Si se da esa correspondencia, los datos de encuestas sobre peso al nacer pueden usarse para mejorar las estimaciones de la incidencia nacional de BPN.

La OMS define el bajo peso al nacer como $<2500$ g. Es corriente agrupar los datos sobre peso al nacer en múltiplos de $500 \mathrm{~g}$, lo cual afecta la estimación de la incidencia de BPN. Por esa razón, en el presente estudio se examinó el grado de agrupamiento en los $2500 \mathrm{~g}$ de las encuestas del programa DHS. En los análisis generales por país de ese estudio, la mitad de los pesos al nacer anotados como $2500 \mathrm{~g}$ se clasificaron BPN.

Además, nuestro análisis enfoca la utilidad de las categorías "pequeño(a)" o "muy pequeño(a)" para indicar el BPN. La magnitud del sesgo de disclasificación se calculó analizando la sensibilidad y el valor predictivo positivo (VPP) del tamaño al nacer como indicador de BPN. Cuando el sesgo de disclasificación fue pequeño, la proporción de recién nacidos clasificados de "pequeños(as)" o "muy pequeños(as)" pudo usarse como indicador de la incidencia de BPN. Aun más importante, los datos sobre tamaño al nacer pudieron usarse para análisis de escala individual sobre los determinantes y las consecuencias del BPN.

\section{RESULTADOS}

El cuadro 1 muestra los países incluidos en las encuestas del programa DHS, el año en que se realizó cada una y el número de nacimientos durante los 5 años anteriores. Hubo una gran variación entre encuestas en 
CUADRO 1. Características de las encuestas y distribución de nacimientos según la disponibilidad de datos sobre peso al nacer

\begin{tabular}{|c|c|c|c|c|c|c|c|}
\hline & \multirow[b]{2}{*}{ Año } & \multirow[b]{2}{*}{$\begin{array}{l}\text { Nacimientos } \\
{(\text { No. })^{\mathrm{a}}}\end{array}$} & \multirow[b]{2}{*}{$\begin{array}{l}\% \text { pesados } \\
\text { al nacer }\end{array}$} & \multicolumn{4}{|c|}{$\begin{array}{c}\% \text { de nacimientos con peso } \\
\text { o tamaño relatado }\end{array}$} \\
\hline & & & & $\begin{array}{l}\text { Solo } \\
\text { peso }\end{array}$ & $\begin{array}{l}\text { Solo } \\
\text { tamaño }\end{array}$ & $\begin{array}{l}\text { Ambos } \\
\text { faltaron }\end{array}$ & Total \\
\hline \multicolumn{8}{|l|}{ África Subsahariana } \\
\hline Camerún & 1991 & 3350 & 61,1 & 50,7 & 49,2 & 0,1 & 100,0 \\
\hline Namibia & 1992 & 3966 & 71,6 & 44,3 & 54,0 & 1,8 & 100,0 \\
\hline Zambia & 1992 & 6479 & 50,5 & 41,9 & 57,8 & 0,3 & 100,0 \\
\hline \multicolumn{8}{|c|}{ Norte de África y Mediterráneo Oriental } \\
\hline Jordania & 1991 & 8364 & $N D^{b}$ & 85,7 & 13,9 & 0,4 & 100,0 \\
\hline Marruecos & 1992 & 5245 & 29,5 & 21,6 & 78,3 & 0,1 & 100,0 \\
\hline Yemen & $1991-2$ & 7230 & 8,8 & 5,8 & 73,3 & 20,9 & 100,0 \\
\hline Colombia & 1990 & 3751 & 80,7 & 62,2 & 37,5 & 0,3 & 100,0 \\
\hline Paraguay & $1991-2$ & 4246 & 72,5 & 71,7 & 27,7 & 0,6 & 100,0 \\
\hline Perú & 1991 & 9461 & 64,2 & 62,0 & 37,6 & 0,4 & 100,0 \\
\hline República Dominicana & 1991 & 4164 & 90,7 & 90,3 & 9,5 & 0,2 & 100,0 \\
\hline
\end{tabular}

a Número de nacimientos durante los 5 años anteriores a la encuesta.

${ }^{\mathrm{b}} \mathrm{ND}=$ no disponible.

cuanto a la proporción de niños que fueron pesados al nacer: desde $9 \%$ en Pakistán y Yemen hasta 91\% en la República Dominicana. La proporción de niños para los cuales había un peso numérico anotado varió desde $6 \%$ en Yemen hasta 90\% en la República Dominicana. La mayoría de las muje-

CUADRO 2. Unidades empleadas en los registros de peso al nacer y agrupamiento por múltiplos de $500 \mathrm{~g}$ y en los $2500 \mathrm{~g}$, programa de Encuestas Demográficas y de Salud (DHS)

\begin{tabular}{lccc}
\hline \multicolumn{1}{c}{ Encuesta } & Unidad & $\begin{array}{c}\text { Agrupamiento por } \\
\text { múltiplos de } 500 \mathrm{~g}^{\mathrm{b}}\end{array}$ & $\begin{array}{c}\text { Agrupamiento } \\
\text { en los } 2500 \mathrm{~g}^{\mathrm{c}}\end{array}$ \\
\hline Camerún & $\mathrm{g}$ & 33,6 & 4,9 \\
Namibia & $\mathrm{g}$ & 22,2 & 2,9 \\
Níger & $\mathrm{kg} 1$ & 29,6 & 8,6 \\
Nigeria & $\mathrm{kg} 1$ & 44,1 & 6,7 \\
Tanzanía & $\mathrm{kg} 2$ & 46,6 & 9,1 \\
Zambia & $\mathrm{kg} 1$ & 31,8 & 6,5 \\
& & & 6,8 \\
Jordania & $\mathrm{g}$ & 31,6 & 5,7 \\
Marruecos & $\mathrm{kg} 1$ & 49,2 & 23,5 \\
Yemen & $\mathrm{g}$ & 54,7 & 6,2 \\
& $\mathrm{~g}$ & 37,7 & 0,2 \\
Indonesia & $\mathrm{g} / \mathrm{g}$ & 0,6 & 5,7 \\
Pakistán & $\mathrm{g}$ & 51,3 & 2,7 \\
Colombia & $\mathrm{g}$ & 30,5 & 3,7 \\
Paraguay & $\mathrm{kg} 1$ & 29,2 & 0 \\
Perú & $\mathrm{lbs}$ & 0 & \\
República Dominicana & & &
\end{tabular}

agn = kilogramos con $n$ decimales; lbs = libras con onzas.

b Porcentaje de todos los pesos relatados en múltiplos de $500 \mathrm{~g}$.

c Porcentaje de todos los pesos agrupados en los $2500 \mathrm{~g}$.

res que declararon que su niño se había pesado recordaban el peso. En 10 de 14 encuestas, más de tres cuartas partes de las madres que dijeron que sus hijos habían sido pesados recordaron el peso. Sin embargo, en Nigeria solo $36 \%$ de las madres que declararon que su niño se había pesado informaron del peso al entrevistador. En Jordania, el peso relatado se anotó sin antes preguntar si alguien había pesado al neonato.

El cuadro 2 muestra las unidades de medición usadas para registrar los pesos al nacer y la frecuencia con que las respuestas se agruparon en múltiplos de $500 \mathrm{~g}$. En siete encuestas, los datos se registraron en g; en cinco, en $\mathrm{kg}$ llevados hasta un lugar decimal; $\mathrm{y}$ en una, en $\mathrm{kg}$ con dos lugares decimales. En la República Dominicana los pesos se registraron en libras y onzas, mientras que en Pakistán se anotaron unas veces en libras y otras, en kilogramos.

En las encuestas con pesos registrados en $\mathrm{g}$ o kg hubo agrupamiento considerable y entre un tercio y la mitad de las respuestas estaban en múltiplos de 500 g. Este agrupamiento fue espe- 
cialmente inconveniente a los $2500 \mathrm{~g}$, es decir, en el punto límite de BPN. De las encuestas con registros en $\mathrm{kg} \mathrm{o} \mathrm{g}$, solo cinco países tuvieron menos de $5 \%$ de las respuestas agrupadas en múltiplos de $500 \mathrm{~g}$ y ocho países tuvieron de 5 a 9\% agrupadas en los 2500 g. En Yemen, casi una cuarta parte de todas las respuestas estaban agrupadas en los 2500 g. En la República Dominicana y Pakistán, 42 y $85 \%$, respectivamente, de las respuestas estuvieron agrupadas de forma similar en múltiplos de libras enteras. El grado de agrupamiento no pareció estar relacionado con la unidad de medición usada.

La distribución del tamaño relativo al nacer recordado aparece en el cuadro 3 con las respuestas divididas entre si hubo o no relato de un peso numérico. La distribución del tamaño relativo varió mucho entre las encuestas; por ejemplo, la proporción notificada como correspondiente al "término medio" osciló de $29 \%$ en la República Dominicana a $79,8 \%$ en la República Unida de Tanzanía. En Yemen la distribución del peso relativo al nacer se diferencia de las demás encuestas en que casi todas las respuestas se agruparon en dos categorías: "de término medio" y "muy pequeño(a)".

Dentro de los países, la distribución del peso relativo al nacer también se diferencia según si hubo o no un peso numérico registrado. En todos los países, la distribución se desplazó hacia los tamaños más pequeños si no había peso numérico registrado. En todas las encuestas excepto la de Zambia, la diferencia entre las dos distribuciones fue significativa (prueba de KolmogorovSmirnov, $P<0$,05).

Como se indicó antes, la calidad de los datos sobre el tamaño relativo al nacer solo puede evaluarse en los niños con pesos numéricos registrados. Los cuadros 4 y 5 presentan datos sobre esos niños exclusivamente. El cuadro 4 enseña la media del peso al nacer en cada categoría de tamaño, la desviación estándar (DE), el coeficiente de variación y el número de $\mathrm{DE}$ con que la media de cada categoría se diferencia de la media general de cada encuesta en particular. La media del peso al nacer varió mucho por categoría de tamaño al nacer y tuvo la con-

CUADRO 3. Distribución porcentual del tamaño al nacer según el peso numérico recordado, encuestas del DHS

\begin{tabular}{|c|c|c|c|c|c|c|c|c|c|}
\hline \multirow[b]{2}{*}{ Encuesta } & \multirow[b]{2}{*}{$\begin{array}{c}\text { Peso } \\
\text { numérico } \\
\text { relatado }\end{array}$} & \multicolumn{6}{|c|}{ Tamaño al nacer } & \multirow[b]{2}{*}{$\begin{array}{l}\text { Total } \\
(\%)\end{array}$} & \multirow[b]{2}{*}{$\begin{array}{c}\text { Nacimientos } \\
\text { (No.) }\end{array}$} \\
\hline & & $\begin{array}{c}\text { Muy } \\
\text { grande } \\
(\%)\end{array}$ & $\begin{array}{l}\text { Grande } \\
\text { (\%) }\end{array}$ & $\begin{array}{c}\text { De término } \\
\text { medio } \\
(\%)\end{array}$ & $\begin{array}{c}\text { Pequeño(a) } \\
(\%)\end{array}$ & $\begin{array}{c}\text { Muy } \\
\text { pequeño(a) } \\
(\%)\end{array}$ & $\begin{array}{c}\text { Faltantes } \\
(\%)\end{array}$ & & \\
\hline \multirow[t]{2}{*}{ Camerún } & Sí & $N^{a}$ & 38,7 & 49,7 & 9,0 & 2,5 & 0,2 & 100,0 & 1699 \\
\hline & No & ND & 26,6 & 54,4 & 16,0 & 2,9 & 0,1 & 100,0 & 1649 \\
\hline \multirow[t]{2}{*}{ Namibia } & Sí & 6,3 & 5,5 & 72,3 & 10,5 & 5,3 & 0,2 & 100,0 & 1755 \\
\hline & No & 6,4 & 4,7 & 66,3 & 9,8 & 9,6 & 3,2 & 100,0 & 2140 \\
\hline \multirow[t]{2}{*}{ Níger } & Sí & 8,2 & 26,8 & 40,2 & 17,4 & 5,9 & 1,6 & 100,0 & 875 \\
\hline & No & 5,3 & 14,4 & 40,6 & 22,2 & 16,0 & 1,3 & 100,0 & 4357 \\
\hline \multirow[t]{2}{*}{ Nigeria } & Sí & 16,0 & 18,5 & 54,3 & 7,4 & 3,6 & 0,3 & 100,0 & 752 \\
\hline & No & 16,7 & 12,5 & 52,1 & 10,3 & 6,7 & 1,8 & 100,0 & 7020 \\
\hline \multirow[t]{2}{*}{ Tanzanía } & Sí & 1,8 & 10,2 & 78,7 & 7,0 & 2,1 & 0,3 & 100,0 & 4015 \\
\hline & No & 1,3 & 6,7 & 80,8 & 7,1 & 2,6 & 1,5 & 100,0 & 4060 \\
\hline \multirow[t]{2}{*}{ Zambia } & Sí & 4,5 & 15,3 & 68,0 & 9,4 & 2,6 & 0,3 & 100,0 & 2792 \\
\hline & No & 2,3 & 17,5 & 68,3 & 9,4 & 2,0 & 0,5 & 100,0 & 3666 \\
\hline \multirow[t]{2}{*}{ Jordania } & Sí & 2,5 & 12,4 & 68,4 & 10,2 & 6,4 & 0,2 & 100,0 & 7169 \\
\hline & No & 0,8 & 6,7 & 73,4 & 8,7 & 7,6 & 2,7 & 100,0 & 1163 \\
\hline \multirow[t]{2}{*}{ Marruecos } & Sí & 3,0 & 24,1 & 53,8 & 14,8 & 4,3 & 0,0 & 100,0 & 1133 \\
\hline & No & 1,3 & 18,0 & 52,8 & 23,6 & 4,2 & 0,1 & 100,0 & 4108 \\
\hline \multirow[t]{2}{*}{ Yemen } & Sí & ND & 2,9 & 87,0 & 0,4 & 8,8 & 0,9 & 100,0 & 421 \\
\hline & No & ND & 1,6 & 63,1 & 0,3 & 12,9 & 22,2 & 100,0 & 3894 \\
\hline \multirow[t]{2}{*}{ Indonesia } & Sí & 8,5 & 29,8 & 49,4 & 10,3 & 1,9 & 0,1 & 100,0 & 5787 \\
\hline & No & 4,2 & 21,2 & 59,8 & 11,7 & 1,4 & 1,8 & 100,0 & 9739 \\
\hline \multirow[t]{2}{*}{ Pakistán } & Sí & 2,0 & 16,6 & 64,3 & 11,6 & 5,4 & 0,0 & 100,0 & 477 \\
\hline & No & 1,9 & 9,1 & 64,6 & 16,4 & 6,4 & 1,6 & 100,0 & 5850 \\
\hline \multirow[t]{2}{*}{ Colombia } & Sí & 8,9 & 23,6 & 49,3 & 10,9 & 7,2 & 0,2 & 100,0 & 2334 \\
\hline & No & 4,1 & 24,9 & 47,5 & 13,7 & 8,9 & 1,0 & 100,0 & 1405 \\
\hline \multirow[t]{2}{*}{ Paraguay } & Sí & ND & 35,0 & 47,3 & 17,3 & ND & 0,3 & 100,0 & 3045 \\
\hline & No & ND & 34,6 & 37,1 & 26,2 & ND & 2,1 & 100,0 & 1364 \\
\hline \multirow[t]{2}{*}{ Perú } & Sí & 1,8 & 22,4 & 57,9 & 14,1 & 3,5 & 0,3 & 100,0 & 5867 \\
\hline & No & 0,7 & 15,7 & 57,9 & 20,5 & 4,2 & 1,0 & 100,0 & 3558 \\
\hline \multirow[t]{2}{*}{ República Dominicana } & Sí & 4,7 & 47,0 & 29,1 & 16,0 & 3,2 & 0,1 & 100,0 & 3761 \\
\hline & No & 3,1 & 39,6 & 26,9 & 22,1 & 6,3 & 2,1 & 100,0 & 394 \\
\hline
\end{tabular}

${ }^{a} \mathrm{ND}=$ no disponible. 
CUADRO 4. Distribución de la media del peso al nacer con desviación estándar (DE), coeficiente de variación (CV) y desviación estándar de la media general, encuestas del DHS

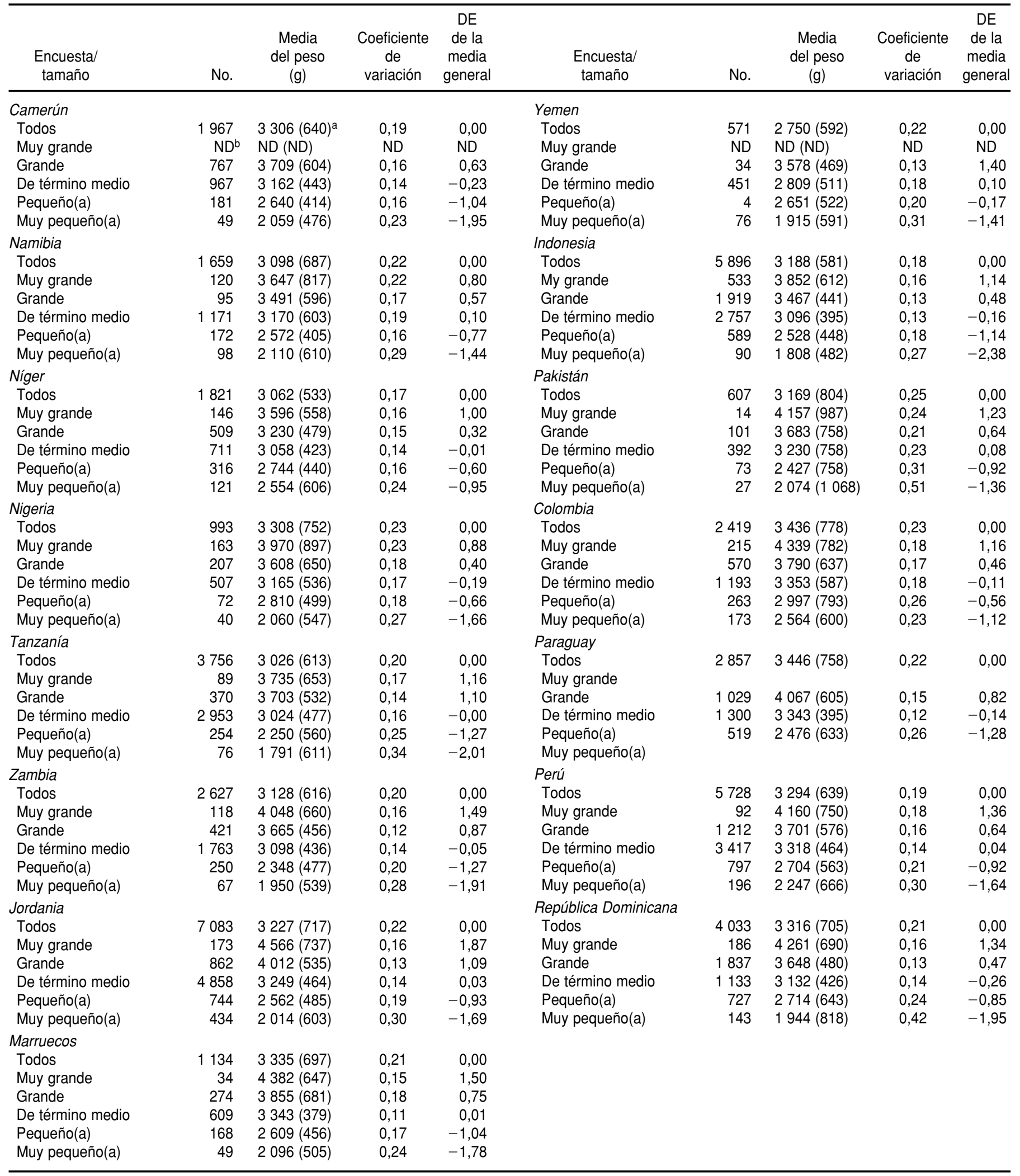

a Las cifras en paréntesis son desviaciones estándar (DE).

${ }^{\mathrm{b}} \mathrm{ND}=$ no disponible. 
CUADRO 5. Sensibilidad, valor predictivo positivo (VPP), especificidad y valor predictivo negativo (VPN) de los tamaños relativos "muy pequeño(a)" y "pequeño(a)" como indicadores de bajo peso al nacer

\begin{tabular}{|c|c|c|c|c|c|c|c|c|}
\hline \multirow[b]{2}{*}{ Encuesta } & \multicolumn{4}{|c|}{ Muy pequeño(a) } & \multicolumn{4}{|c|}{ Pequeño(a) } \\
\hline & $\begin{array}{c}\text { Sensibilidad }{ }^{\mathrm{b}} \\
(\%)\end{array}$ & $\begin{array}{l}\text { VPP } \\
(\%)\end{array}$ & $\begin{array}{c}\text { Especificidad } \\
(\%)\end{array}$ & $\begin{array}{l}\text { VPN } \\
(\%)\end{array}$ & $\begin{array}{c}\text { Sensibilidad } \\
(\%)\end{array}$ & $\begin{array}{l}\text { VPP } \\
(\%)\end{array}$ & $\begin{array}{c}\text { Especificidad } \\
(\%)\end{array}$ & $\begin{array}{l}\text { VPN } \\
(\%)\end{array}$ \\
\hline Camerún & 29 & 72 & 99 & 95 & 62 & 33 & 97 & 97 \\
\hline Namibia & 30 & 70 & 98 & 91 & 60 & 47 & 91 & 94 \\
\hline Níger & 24 & 35 & 96 & 93 & 61 & 23 & 80 & 96 \\
\hline Nigeria & 31 & 63 & 99 & 95 & 46 & 31 & 92 & 96 \\
\hline Tanzanía & 13 & 86 & 100 & 88 & 46 & 69 & 97 & 92 \\
\hline Zambia & 20 & 89 & 100 & 91 & 68 & 65 & 95 & 96 \\
\hline Jordania & 45 & 71 & 98 & 94 & 75 & 46 & 90 & 97 \\
\hline Marruecos & 36 & 63 & 98 & 95 & 90 & 35 & 87 & 99 \\
\hline Yemen & 31 & 73 & 97 & 84 & 32 & 71 & 97 & 84 \\
\hline Indonesia & 23 & 88 & 100 & 94 & 71 & 42 & 92 & 98 \\
\hline Pakistán & 29 & 85 & 99 & 88 & 68 & 64 & 93 & 94 \\
\hline Colombia & 55 & 37 & 95 & 97 & 83 & 23 & 85 & 99 \\
\hline Paraguay & & & & & 87 & 41 & 89 & 99 \\
\hline Perú & 23 & 66 & 99 & 96 & 41 & 70 & 88 & 97 \\
\hline República & & & & & & & & \\
\hline Dominicana & 24 & 81 & 99 & 91 & 78 & 46 & 88 & 97 \\
\hline Promedio & 29 & 70 & 98 & 92 & 66 & 45 & 91 & 96 \\
\hline
\end{tabular}

a Incluye la categoría "muy pequeño(a)".

b Proporción de recién nacidos con bajo peso al nacer detectados por el indicador del tamaño relativo.

c Proporción de recién nacidos sin bajo peso al nacer incluidos en las categorías "muy pequeño(a)" y "pequeño(a)".

figuración esperada en todos los países, es decir, que la media más baja correspondió a la categoría "muy pequeño(a)" la segunda más baja a la categoría "pequeño(a)" y así sucesivamente.

Excepto en Colombia y Nigeria, la media de los pesos al nacer de los bebés muy pequeños estuvo muy por debajo del punto límite del PBN y se ubicó dentro de un recorrido de 1800 a $2300 \mathrm{~g}$. En 11 de 14 encuestas, la media de los bebés muy pequeños cayó de 1,4 a 2 unidades de DE por debajo de la media general. El coeficiente de variación más alto correspondió a la categoría "muy pequeño(a)" de todas las encuestas menos la de Colombia, lo cual muestra cuánto puede variar lo que se entiende por "muy pequeño(a)".

La media del peso al nacer de los niños registrados como "muy grandes" fue de $>3500 \mathrm{~g}$ en todas las encuestas y $>4000$ g en 7 de 12 encuestas. La media del peso de la categoría "muy grande" se ubicó entre 1 y 1,5 DE por encima de la media del peso al nacer de la mayor parte de las encuestas.
La proporción de bebés recordados como "muy pequeño(a)s" o "pequeño(a)s" al nacer, ¿puede utilizarse como indicador de la proporción de bebés con BPN? El cuadro 5 muestra la sensibilidad, VPP y especificidad de tamaño relativo al nacer como indicador de BPN. Aquí la sensibilidad debe entenderse como la proporción de neonatos de bajo peso reconocidos por el indicador de tamaño relativo al nacer; el VPP, como la proporción de neonatos de bajo peso entre los reconocidos por el indicador de tamaño relativo al nacer; y la especificidad, como la proporción de neonatos que no eran de bajo peso entre los que se incluyeron en las categorías de "muy pequeño(a)s" o "pequeño(a)s". La sensibilidad de la categoría de tamaño relativo al nacer fue muy baja en todas las encuestas (media, 29\%) si bien el VPP fue más de $70 \%$ en la mayor parte de las encuestas. Así que la mayoría de los bebés recordados como muy pequeños son en realidad de bajo peso al nacer, pero solamente alrededor de un cuarto o un tercio de todos los de bajo peso están incluidos en esta categoría.

La sensibilidad mejora mucho cuando se usan las dos clasificaciones de muy pequeño y pequeño como indicadores de BPN (véase el cuadro 5). Sin embargo, mientras que las dos medidas de tamaño relativo más pequeñas comprenden dos tercios de los bebés con BPN (media de sensibilidad, 66\%), los neonatos recordados como pequeños o muy pequeños son menos propensos a tener BPN. En 11 de 15 encuestas, menos de $50 \%$ de los muy pequeños o pequeños tuvieron BPN (media del VPP, 45\%).

El cuadro 6 muestra las estimaciones de la media del peso al nacer y de la incidencia de BPN, primero de nacimientos con pesos registrados, seguidos de nacimientos con datos de tamaño solamente y por último de todos los nacimientos. Los pesos al nacer estimados solo a base de datos de tamaño relativo se calcularon usando la media del peso al nacer de cada categoría de tamaño de entre los neonatos de peso numérico conocido y la distribución de tamaños de los que no tenían peso numérico conocido.

Tal como podría esperarse sobre la base de la distribución de tamaños que aparece en el cuadro 3, la incidencia de BPN fue más alta cuando se tuvieron en cuenta todos los nacimientos que cuando solo se incluyeron aquellos con datos numéricos. En ocho países, la incidencia de BPN aumentó más de $1 \%$, y en Níger, Nigeria, Pakistán y Marruecos, mucho más de $2 \%$. Los datos de Yemen son muy distintos de los de las otras encuestas, tal vez debido a mala calidad.

\section{DISCUSIÓN}

Las encuestas retrospectivas pueden mejorar las estimaciones de la incidencia de BPN en países en desarrollo, donde no se pesa a una gran parte de los recién nacidos. La evaluación de la calidad de los datos sobre el peso al nacer indicó que los datos de tamaño relativo al nacer son indicadores razonablemente buenos del peso al nacer a escala general. No obstante, deben 
CUADRO 6. Media del peso al nacer e incidencia de bajo peso al nacer (BPN) entre niños con peso numérico relatado, niños con tamaño relativo recordado solamente y todos los niños

\begin{tabular}{|c|c|c|c|c|c|c|}
\hline & \multicolumn{2}{|c|}{ Peso numérico solamente } & \multicolumn{2}{|c|}{ Tamaño solamente } & \multicolumn{2}{|c|}{ Todos } \\
\hline & $\begin{array}{l}\text { Media } \\
\text { (g) }\end{array}$ & $\begin{array}{l}\text { BPN } \\
(\%)\end{array}$ & $\begin{array}{l}\text { Media } \\
\text { (g) }\end{array}$ & $\begin{array}{l}\text { BPN } \\
(\%)\end{array}$ & $\begin{array}{c}\text { Media } \\
\text { (g) }\end{array}$ & $\begin{array}{l}\text { BPN } \\
(\%)\end{array}$ \\
\hline Camerún & 3302 & 8,6 & 3193 & 11,6 & 3248 & 10,1 \\
\hline Namibia & 3098 & 13,9 & 3042 & 17,3 & 3067 & 15,8 \\
\hline Níger & 3062 & 13,3 & 2958 & 17,9 & 2971 & 17,3 \\
\hline Nigeria & 3308 & 10,9 & 3232 & 14,1 & 3240 & 13,8 \\
\hline Tanzanía & 3024 & 18,4 & 2992 & 19,2 & 3008 & 18,8 \\
\hline Zambia & 3128 & 14,6 & 3126 & 14,2 & 3127 & 14,4 \\
\hline Jordania & 3228 & 13,6 & 3154 & 14,9 & 3218 & 13,8 \\
\hline Marruecos & 3335 & 10,4 & 3219 & 13,7 & 3244 & 13,0 \\
\hline Yemen & 2751 & 32,8 & 2253 & 29,7 & - a & $-\mathrm{a}$ \\
\hline Indonesia & 3188 & 10,3 & 3118 & 11 & 3144 & 10,7 \\
\hline Pakistán & 3169 & 16,1 & 3030 & 19,3 & 3041 & 19,0 \\
\hline Colombia & 3436 & 8,3 & 3374 & 9,5 & 3413 & 8,7 \\
\hline Paraguay & 3446 & 9,5 & 3366 & 13,4 & 3424 & 10,6 \\
\hline Perú & 3294 & 10,2 & 3211 & 12,7 & 3263 & 11,2 \\
\hline República Dominicana & 3316 & 11,7 & 3210 & 16,9 & 3313 & 11,8 \\
\hline
\end{tabular}

a No se estimó.

tenerse en cuenta dos cosas. Primero, que la calidad de los datos sobre el tamaño relativo al nacer puede evaluarse solamente en los niños cuyo peso numérico se conoce. Hemos supuesto que los datos sobre el tamaño relativo al nacer de los niños cuyo peso numérico no se conoce son de igual calidad que los de los niños con peso numérico conocido. Sin embargo, es posible que las madres que recuerdan los pesos al nacer numéricos puedan evaluar mejor el tamaño relativo al nacer que otras madres cuyos niños no se pesaron al nacer. Probablemente los datos sobre el tamaño relativo al nacer de niños cuyo peso numérico no se registró sean de menor calidad que los de niños cuyo peso numérico fue registrado. Segundo, la calidad de los datos numéricos del peso al nacer no puede evaluarse con detalle. Se encontró mucha agrupación de los datos, lo cual puede ser el resultado de errores de recuerdo. Sin embargo, los datos mantenidos en instalaciones de salud están igualmente sembrados de múltiplos de $500 \mathrm{~g}$, lo que indica un frecuente redondeo del peso.

En la mayoría de los países considerados en este estudio, no hay estima- ciones nacionales de la incidencia de BPN, basadas en centros de atención sanitaria. Solamente la República Unida de Tanzanía tenía un número suficiente de estudios del peso al nacer en el banco de datos de la OMS que permitieron compararlos con los datos de los centros de atención de salud. ${ }^{6}$ Datos de 1975 a 1986 sobre más de 100000 nacimientos en 16 diferentes localidades muestran una media de peso al nacer apenas bajo los $3000 \mathrm{~g}$ y una incidencia de BPN de $14 \%$. La media se aproxima bastante a la estimación de 3024 aquí presentada. Aun así, es posible que la incidencia menor de BPN registrada en los centros de atención de salud sea en parte el resultado de haber excluido de la categoría de BPN a todos los niños de $2500 \mathrm{~g}$ (en nuestro estudio, la mitad de esos niños se consideraron de BPN).

Ya que nosotros asignamos pesos a los niños para quienes solo había datos de tamaño, las estimaciones generales de peso al nacer aumentan en incerti-

\footnotetext{
6 Organización Mundial de la Salud. Low birth weight: a tabulation of available information. Ginebra, 1992. (Documento inédito WHO/MCH/ 92.2.)
}

tud a medida que disminuye la proporción de niños con peso numérico registrado. En el caso de Yemen, no se estimó la incidencia de BPN, porque el peso numérico registrado correspondía a solo $6 \%$ de los nacimientos y, además, porque en general los datos eran de mala calidad. Una cuarta parte de las respuestas numéricas estaban agrupadas en los $2500 \mathrm{~g}$, no había datos de peso ni tamaño relativo al nacer para una quinta parte de los niños registrados en la encuesta y los tamaños relativos recordados estaban agrupados en dos categorías solamente. Por lo tanto, se recomienda que el tamaño relativo no se use para estimar el BPN en encuestas con categorías de "pequeño(a)" y "muy pequeño(a)" que tienen menos de 25 niños para quienes se dispone también de medidas numéricas de peso al nacer. Si el número de estos es de 25 a 50, las estimaciones deben interpretarse con cautela. En consecuencia, las estimaciones para países como Nigeria y Pakistán, donde se registraron pesos numéricos de $<50$ neonatos, son menos precisas que las de países donde se registraron datos numéricos de por lo menos $40 \%$ de los nacimientos (9 de 15 países). Sin embargo, es difícil cuantificar el punto en que los nacimientos son demasiado escasos para producir una estimación general confiable de la incidencia de BPN. Además, si el grupo que informa del peso al nacer se limita principalmente a un subgrupo en particular (mujeres pudientes de centros urbanos, por ejemplo) no pueden hacerse estimaciones nacionales de la incidencia de BPN.

Debido a que la disclasificación del peso al nacer es tan frecuente, la categoría de "muy pequeño(a)"no ha resultado útil como indicador de un peso menor de $2500 \mathrm{~g}$ en los análisis de escala individual sobre los determinantes del BPN y los efectos de este en la mortalidad infantil. No obstante, las encuestas transversales pueden proporcionar una fuente de datos que permite estimar los pesos al nacer y la incidencia de BPN a escala nacional $\mathrm{y}$, si la muestra es suficientemente grande, también pueden hacerse estimaciones regionales dentro de un país. 


\section{REFERENCIAS}

1. McCormick MC. The contribution of low birth weight to infant mortality and childhood mortality. New Engl J Med 1985;312: 82-90.

2. Kourguéni IA, et al. Enquête démographique et de santé Niger 1992. Niamey, Niger: Ministère des Finances et du Plan; 1993.

3. Azelman $\mathrm{M}$ et al. Enquête nationale sur la Population et la Santé (ENPS-II) 1992. Rabat. Maroc: Ministère de la Santé Públique; 1993.
4. Pakistan demographic and health survey, 1990/ 1991. Islamabad, Pakistan: National Institute of Population Studies; 1992.

5. Indonesia demographic and health survey 1991. Jakarta, Indonesia: Ministry of Health; 1992.

6. Yemen demographic and maternal and child health survey 1991/1992. Calverton, Maryland, USA: Macro International; 1994.

7. Da Vanzo J, Habicht JP, Butz WP. Assessing socioeconomic correlates of birthweight in peninsular Malaysia: ethnic differences and changes over time. Soci Sci Med 1984; 18: 387-404.

8. Moreno L, Goldman SP. An assessment of survey data on birthweight. Soc Sci Med 1990; 31: 491-500.

9. Ekouevi K, Morgan SP. Note on the reliability and validity of mothers' retrospective reports of their children's birthweights. Soc Biol 1991; 38:140-145.
ABSTRACT

\section{Data on birth weight in developing countries: can surveys help?}

The main source of data on birth weight in developing countries is statistics from health facilities, although most developing countries do not produce annual estimates of the incidence of low birth weight from these data. Such estimates would be subject to selection bias as the data are usually limited to babies born within health facilities, and therefore are representative of a subgroup that is markedly different from the overall population of neonates. Since 1990 the Demographic and Health Surveys programme has included questions on recalled birth weight and relative size at birth in 15 national surveys. In this article, we show that these cross-sectional surveys can provide a useful data source for making national estimates of mean birth weight and the incidence of low birth weight. The extent of misclassification of birth weight is, however, too large to use the data on relative size as birth as an indicator of low birth weight at the individual level. 